DR ETIENNE CHALLET (Orcid ID : 0000-0001-9416-9496)

DR PAUL PÉVET (Orcid ID : 0000-0002-8471-3619)

DR KHALID EL ALLALI (Orcid ID : 0000-0002-6897-4835)

Article type : Original Manuscript

\title{
Melatonin rhythm and other outputs of the master circadian clock in the desert goat (Capra hircus) are entrained by daily cycles of ambient temperature
}

Hicham Farsi ${ }^{1}$, Driss Harti ${ }^{1}$, Mohamed Rachid Achaâban ${ }^{1}$, Mohammed Piro ${ }^{2}$, Véronique Raverot ${ }^{4}$, Béatrice Bothorel ${ }^{3}$, Mohammed Ouassat ${ }^{1}$, Etienne Challet ${ }^{3}$, Paul Pévet ${ }^{3}$ and Khalid El Allali ${ }^{1}$.

1. Comparative Anatomy Unit, Department of Biological and Pharmaceutical Veterinary Sciences, Hassan II Agronomy and Veterinary Medicine Institute, Rabat, Morocco.

2. Medicine and Surgical Unit of domestic animals, Department of Medicine, Surgery and reproduction, Hassan II Agronomy and Veterinary Medicine Institute, Rabat, Morocco.

3. Institute of Cellular and Integrative Neurosciences, CNRS and University of Strasbourg, Strasbourg, France.

4. Laboratory of Hormonology, East Center for Biology and Pathology, East Hospital Group, Civil Hospices of Lyon, France.

Running title: Ambient temperature cycle as a zeitgeber in goat.

This article has been accepted for publication and undergone full peer review but has not been through the copyediting, typesetting, pagination and proofreading process, which may lead to differences between this version and the Version of Record. Please cite this article as doi: 10.1111/jpi.12634

This article is protected by copyright. All rights reserved 


\section{Corresponding author:}

Khalid El Allali,

Comparative Anatomy Unit, Department of Biological and Pharmaceutical Veterinary Sciences, Hassan II Agronomy and Veterinary Institute, BP: 6202, 10101 Rabat-Instituts, Rabat-Morocco. Phone: +212661140470

E-mail: khalid_elallali@yahoo.fr

\section{Abstract}

In desert areas, mammals such as camel and goat are exposed to harsh environmental conditions. The ambient temperature ( $\mathrm{Ta}$ ) cycles have been shown to entrain the circadian clock in the camel. In the present work, we assumed that, in the goat living in a desert biotope, Ta cycles would have the same synchronizing effect on the central clock. Therefore, the effects of Ta cycles on body temperature ( $\mathrm{Tb})$, locomotor activity (LA) and melatonin (Mel) rhythms as outputs of the master circadian clock have been studied. The study was performed on bucks kept first under constant conditions of total darkness (DD) and constant Ta, then maintained under DD conditions but exposed to Ta cycles with heat period during subjective day and cold period during subjective night. Finally, the Ta cycles were reversed with highest temperatures during the subjective night and the lowest temperatures during the subjective day. Under constant conditions, the circadian rhythms of $\mathrm{Tb}$ and LA were free-running with an endogenous period of 25.3 and $25.0 \mathrm{~h}$, respectively. Ta cycles entrained the rhythms of $\mathrm{Tb}$ and LA to a period of exactly 24.0h; while, when reversed, the Ta cycles led to an inversion of Tb and LA rhythms. Similarly, Ta cycles were also able to entrain Mel rhythm, by adjusting its secretion to the cooling phase before and after Ta

This article is protected by copyright. All rights reserved 
cycles inversion. All together, these results show that the Ta cycles entrain the master circadian clock in the goat.

Keys word: Goat, entrainment, body temperature, melatonin, locomotor activity, ambient temperature, circadian clock.

\section{Introduction}

In mammals, the circadian organization of physiological and behavioural functions is under the control of the master clock, located within the hypothalamic supra-chiasmatic nuclei (SCN), and of secondary clocks within peripheral organs ${ }^{1,2,3}$. The light-dark (LD) cycle was found to be the most powerful zeitgeber for the SCN (For review see ${ }^{2,3}$ ). How ambient light synchronizes the circadian system is now well documented ${ }^{4,5,6,7}$. However, little is known about the ability of ambient temperature (Ta) cycles to entrain circadian rhythms. Such entrainment is well established for the circadian system in non-mammalian vertebrates (For review see ${ }^{8}$ ). In laboratory mammals, despite the responsiveness of peripheral clocks to the synchronizing effects of ambient temperature cycles $9,10,11$, experiments showed either a complete absence of entrainment of the master clock in some species ${ }^{11,12}$ or a partial entrainment of locomotor activity (LA) rhythm in only some individuals ${ }^{13,14,15,16,17,18}$. In such conditions, it would be very difficult to conclude of a real synchronization of the master circadian clock since, as reported ${ }^{15,16,17,18}$, such entrainment of LA rhythm could be a result of a masking effect of Ta. Accordingly, Ta cycles are assumed to have a weak effect on the master circadian clock at least in endothermic mammals (For review see ${ }^{8}$ ). Within mammals, a group of several species including camel, display heterothermy phenomenon, which means that under special conditions (e.g. dehydration and exposure to high $\mathrm{Ta}$ ), $\mathrm{Tb}$ can follow $\mathrm{Ta}^{19}$. Moreover, it should be noted that the circadian system of heterothermic animals was shown to be more sensitive to environmental $\mathrm{Ta}^{20,21,22}$. Thereby, in a previous study ${ }^{23}$, our team has shown that, in the camel, the Ta cycles are a strong zeitgeber for the circadian clock in the SCN. Two outputs of the master clock, plasma melatonin (Mel) and body temperature (Tb) rhythms were indeed found to be synchronized by Ta cycles. To our knowledge, this finding was the first demonstration in mammals of such non-photic entrainment to Ta.

In camels under heat stress and dehydration, heterothermy corresponds to a switching from a perfect endothermic state to a heterothermic state ${ }^{19,24}$. The Ta synchronization of the circadian 
system described in the camel could be related to this specific thermoregulation process. Recently, such heterothermy has been also shown in other desert ungulates, including oryx and goat ${ }^{25,26,27}$. Thus, the raised question is whether the observed entrainment of the circadian clock by Ta cycles is specific to camel or is a mechanism shared by other desert large species.

To test this hypothesis, we have studied three outputs of the master clock, namely: Tb, LA and Mel rhythms, in the desert goat.

\section{Experimental procedures}

Animals

Seventeen healthy adult male desert goats (Capra hircus) weighing $26 \pm 0.3 \mathrm{~kg}$ and aged around 3.5 years were used in this study. Animals were housed in specific facilities at the Hassan II Agronomy and Veterinary Medicine Institute of Rabat (Latitude: $34^{\circ} 01^{\prime} \mathrm{N}$, Longitude: $6^{\circ} 50^{\prime} \mathrm{W}$ ). Males were used instead of females to avoid eventual effects of oestrous and gestation on circadian rhythms.

This study was carried out in accordance with the International Ethical Standards ${ }^{28}$ and the national recommendations for animal farm husbandry, experimentations and surgeries of the Hassan II Agronomy and Veterinary Medicine Institute of Rabat.

\section{Experimental Design}

Experiment 1 was intended to verify if Ta cycles could entrain the $\mathrm{Tb}$ and $\mathrm{Mel}$ rhythms in the goat. To avoid the masking effect of LA on the Tb rhythm ${ }^{23,29}$, bucks $(n=10)$ were maintained in forced inactivity but allowed an easy standing, moving around and sitting in sternal and lateral recumbency. Animals received a complete compound food (Maraa ${ }^{\circledR}$, Alf-Sahel, Morocco) and hay 4 times a day to avoid a possible entrainment by feeding ${ }^{23,30}$. Water was ad libitum. The air relative humidity during this experiment was around $23 \%$.

This experiment was carried on five stages as follows:

This article is protected by copyright. All rights reserved 
Stage 1 (5 days): is an adaptive period to indoor conditions with an artificial LD cycle (12L-12D) and uncontrolled $\mathrm{Ta}\left(17.00^{\circ} \mathrm{C}-25.50^{\circ} \mathrm{C}\right)$.

Stage 2 (21 days): this step was designed to demonstrate the existence in the goat of a freerunning rhythm of $\mathrm{Tb}$. Animals were placed under constant conditions of total darkness (DD) and constant ambient temperature $(\mathrm{CTa})$ of $23 \pm 0.37^{\circ} \mathrm{C}$ to avoid any temporal perception from the environment. This CTa was chosen as thermo-neutral Ta for goats.

Stage 3 (21 days): the DD conditions were maintained and a 24-h artificial daily Ta cycle (NRT) with a heating-period applied during the subjective day (maximum $31.93^{\circ} \mathrm{C}$, minimum $16.17^{\circ} \mathrm{C}$ ).

Stage 4 (29 days): correspond to the same DD conditions but a 12-h phase advance of Ta cycle (RT) (maximum $32.47^{\circ} \mathrm{C}$, minimum $16.30^{\circ} \mathrm{C}$ ) was realized with a heating-period shifted to occur during the subjective night.

Stage 5 (21 days): a return to the former conditions of stage 3 with Ta cycle peaking under the subjective day (NRTr).

Experiment 2 was conducted on 7 bucks and designed to check if Ta cycles could synchronize the LA rhythm. Each buck was able to move freely in an individual controlled sheltered stable of $36 \mathrm{~m}^{2}$. The same feed diet of experiment 1 and water were provided ad libitum; while the relative humidity was around $21 \%$. To avoid the possible masking effect of the social behaviour of bucks on LA rhythm, experiment was conducted individually and lasts each 125 days. Each individual experiment consisted of the following stages:

Stage 1 (5 days): is an adaptive period to indoor conditions with an artificial LD cycle (12L-12D) and uncontrolled Ta cycle $\left(19.05^{\circ} \mathrm{C}-24.60^{\circ} \mathrm{C}\right)$.

Stage 2 (26 days): This stage was designed to demonstrate the existence in the goat of a freerunning rhythm of LA rhythm. Constant conditions of DD and CTa $\left(23 \pm 0.40^{\circ} \mathrm{C}\right)$ were applied.

Stage 3 (27 days): The DD conditions were maintained and a 24-h artificial Ta cycle (NRT, maximum: $31.38^{\circ} \mathrm{C}$ and minimum: $15.98^{\circ} \mathrm{C}$ ) with a heating-period during the subjective day was applied.

Stage 4 (44 days): Animals were kept under the same conditions of stage 3 but a 12-h phase advance of Ta cycle (RT, maximum $32.68^{\circ} \mathrm{C}$ and minimum $15.01{ }^{\circ} \mathrm{C}$ ) was realized, with a heatingperiod during the subjective night.

This article is protected by copyright. All rights reserved 
Stage 5 (23 days): corresponds to a return to the previous conditions of stage 2.

Details of these experimental procedures are detailed in Figure 1.

\section{Temperature measurements}

The rhythm of $\mathrm{Tb}$ was measured during experiment 1 by using data logger, Ibuttons ${ }^{\circledR}$ (DS1922L, Dallas Semi-Conductor, USA). The devices were programmed for 16-bit with a resolution of $0.0625^{\circ} \mathrm{C}$ and to record $\mathrm{Tb}$ every $12 \mathrm{~min}$. Then, the Ibuttons were protected with silicone elastomer (Rhodorsil RTV141) before their surgical implantation in the abdominal cavity of bucks. This site has been widely used to record $\mathrm{Tb}$ in several species ${ }^{31,32,33}$.

\section{Locomotor activity measurements:}

The LA rhythm of bucks was recorded during experiment 2 using the validated video-locomotion scoring technique (For more details, see $^{34}$ ) which is based on a visual attribution of scores to activity, where 0 : animal on a sitting position and not moving; 1 : animal on a sitting position and moving lightly its limbs beneath the body; 2: animal on standing position without movements; 3 : animal on standing position moving its limbs but without locomotion; 4: animal walking and exploring the environment; 5: animal in intense locomotor activity or agitated.

The LA rhythm was measured separately in each animal using a time set of 5 minutes. This time interval is imposed by the used software which provides activity as actograms. The recorded video sequences were visually analysed on a computer screen by two well-trained evaluators who assigned a score to each activity state (For details, see $^{34}$ ).

\section{Melatonin assay}

To determine the plasma concentration of Mel under various conditions of experiment 1, blood was sampled every 2-hours during the last 24-hours of stages 3, 4 and 5. Blood was collected in 10 $\mathrm{ml}$ lithium heparin BD-Vacutainer tubes by puncturing alternatively the right and left external jugular veins. To minimize stress, bucks were accustomed to handling and sampling procedures for several weeks. The blood sampling was performed under complete darkness without any source of light by well-trained operators. Samples were immediately centrifuged at $1760 \mathrm{~g}$ for 30 min and then plasma was stored at $-20^{\circ} \mathrm{C}$ for subsequent $\mathrm{Mel}$ assay. 
Mel concentrations were measured using an in-house radioimmunoassay after extraction from plasma with diethylether ${ }^{35}$. Duplicated extracted samples were mixed with in-house antibody and labelled 125Iodomelatonin (Perkin Elmer NEX2360), incubated overnight at $4^{\circ} \mathrm{C}$. The radioactivity of the pellet obtained after precipitation by anti-rabbit $\gamma$-globulin solution was counted. The intra-assay CV was $9.5 \%$ at levels 39 and $57 \mathrm{ng} / \mathrm{L}$. The inter-assay CV was $12.7 \%$ at levels $50 \mathrm{ng} / \mathrm{L}$ and $12.4 \%$ at $100 \mathrm{ng} / \mathrm{L}$. The quantification limit was of $3.7 \mathrm{ng} / \mathrm{L}$.

\section{Data Analysis}

Temperature data. Tb data was presented as means \pm Standard Errors of the Means (SEM) and plotted in simple curves using the SigmaPlot ${ }^{\circledR}$ software (Systat. Sigmaplot v12.0, Systat, Chicago, IL). The period, acrophase, mesor, amplitude and the robustness of Tb rhythm were calculated by nonlinear least squares regression using Acro $^{\circledR}$, Cosinor $^{\circledR}$ and LSP $^{\circledR}$ softwares (Refinetti R, Circadian Rhythm Laboratory, University of South Carolina, http://www.circadian.org/softwar.html). Cosinor analysis was performed according to the equation:

\section{$f=y 0+a * \cos [2 \pi *(t-\varphi) / \tau]$}

Where $f$ is Tb at time $t, y 0$ is the mesor, $a$ is the amplitude, $\varphi$ is the acrophase and $\tau$ the period. For each parameter, a confidence interval of $95 \%$ probability is given. Similarly, the degree of significance of the regression is calculated. The One-Way ANOVA test was used to determine if there are statistical differences in the periods and the acrophases of $\mathrm{Tb}$ between the different stages of experiment 1 , mainly between NRT, RT and NRTr conditions.

Locomotor activity data. LA rhythm data was presented as individual actograms using the Actogram Plotter ${ }^{\circledR}$ software (Refinetti R, Circadian Rhythm Laboratory, University of South Carolina, http://www.circadian.org/softwar.html). As for Tb, the rhythm parameters of LA were calculated by the same Cosinor analysis equation.

Similarly, a one-way ANOVA test was also used to compare periods and acrophases of LA rhythm between stages of experiment 2 and also, to verify the equality of means of the daily activity onsets and offsets around the daily Ta transition.

This article is protected by copyright. All rights reserved 
Melatonin data. Results data of Mel were presented as means \pm SEM and plotted using the SigmaPlot ${ }^{\circledR}$ software. The Mel concentrations under different Ta treatments were evaluated separately for each individual and then for the mean of all animals. Accordingly, the Mel rhythm secretion for stages 3, 4 and 5 were fitted using a nonlinear regression of the following exponential mathematical equation $36,37,38$ :

\section{$f=y 0+(y \max /((1+\exp (\operatorname{slope} 1 *(p h i 1-x))) *(1+\exp (\operatorname{slope} 2 *(x-p h i 2)))))$}

Where $\boldsymbol{f}$ is the concentration of Mel variation at time $\boldsymbol{x}, \boldsymbol{y} \boldsymbol{0}$ is the basal level, $\boldsymbol{y} \boldsymbol{m a x}$ is the amplitude of the Mel peak concentration, phil and phi2 are the inflection points and represent the time points at which $50 \%$ of the maximal increase and $50 \%$ of the decline were reached respectively. Slope1 and slope 2 are the slopes of the onset and the offset (inflection points at half the peak height) and were assigned with arbitrary constant values. The equation was chosen because it minimizes the dispersion of the abscissas of the inflection points and their collinearities with other parameters. The algorithm provides an ANOVA for the mathematical regression which was tested using F-test. It also provides an asymptotic estimate (by excess) of the standard error for each parameter. Shapiro-Wilk's test was used to check the homogeneity of residuals and Bartlett's test for the homogeneity of variances ${ }^{23}$. The following step was designed to compare the obtained phil and phi2 between NRT, RT and NRTr conditions of Ta cycles corresponding respectively to stages 3, 4 and 5 of experiment 1. A paired t-test was performed twice to determine the phil and phi2 statistical differences, first between NRT (stage 3) and RT (stage 4) conditions and then between RT (stage 4) and NRTr (stage 5) conditions.

$P \leq 0.05$ was considered statistically significant in all statistical tests.

\section{Results}

Under stage 1 conditions, Tb showed a clear daily rhythm (Figure 2) characterized by a robustness of $40 \pm 2.7 \%$, a mesor of $38.74 \pm 0.12^{\circ} \mathrm{C}$, an amplitude of $0.69 \pm 0.36^{\circ} \mathrm{C}$, an acrophase at $17: 23 \pm 0.28 \mathrm{~h}$ and a period of exactly $24.0 \mathrm{~h}$ (Table 1). Tb was lowest around $08 \mathrm{~h} 00 \mathrm{am}$, started then its ascending phase during the early morning achieving high value in the early evening. After

This article is protected by copyright. All rights reserved 
Light/Dark transition, Tb dropped across the scotophase achieving its lowest value at Dark/Light transition.

Under similar environment (stage 1, experiment 2), LA rhythm showed intense daily activity during the photophase (Figure 3). Cosinor regression of LA rhythm shows that the robustness was of $32.0 \pm 1.8 \%$, the acrophase occurred at the middle of the photophase, exactly at $12: 33 \pm 0.30 \mathrm{~h}$, the period was exactly of $24.0 \mathrm{~h}$ and the mesor and the amplitude were respectively $1.59 \pm 0.08$ and $1.7 \pm 0.09$ (Table 2).

The existence of circadian rhythms in goat was inferred by stage 2 of both experiments 1 and 2 . We observed that both $\mathrm{Tb}$ and LA rhythms (24.0h period) first shown under a cyclic indoor environment of stage 1, persisted when goats were transferred under constant conditions (stage 2) with a period different from 24.0h (Figures 2 and 3; Tables 1 and 2): exactly of $25.3 \pm 0.09 \mathrm{~h}$ for Tb rhythm and $25.0 \pm 0.10 \mathrm{~h}$ for LA rhythm. The one-way-ANOVA showed that the periods of constant

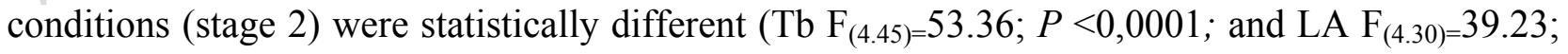
$P<0.0001$ ) from those under indoor cyclic conditions (stage 1).

A slight reduction of the shape regularity of the $\mathrm{Tb}$ and LA curves has been noticed during constant conditions (stage 2: Figures 2 and 3). Moreover, animal activity on double-plot LA actograms appears to be daily shifted indicating a free-running state of LA. In fact, the two rhythms were shifted since acrophases were delayed after transferring animals from cyclic environment to constant conditions (Tables 1 and 2). The acrophase was shifted by $21.5 \mathrm{~h}$ for $\mathrm{Tb}$ and by $22.4 \mathrm{~h}$ for LA during DD and CTa. Under free-running conditions, the robustness also changed, decreasing significantly from $40.0 \%$ to $27.1 \%$ for $\mathrm{Tb}$ and from $32 \%$ to $7.3 \%$ for LA (Tables 1 and 2). These results, together with the persistence of rhythmicity under constant conditions, confirm the existence of a circadian clock that drives these two rhythms.

The next step of this study was to verify if daily Ta cycles are able to synchronize the two outputs of the master circadian clock, the Tb and LA rhythms. This investigation was carried out under DD and Ta cycles of stages 3, 4 and 5 of experiment 1 and stages 3 and 4 of experiment 2. Under DD conditions, the Ta cycles of stage 3 were able to generate regular rhythms of $\mathrm{Tb}$ and LA (Figures 2 and 3). The period changed from 25.3h (Tb) and 25.0h (LA) during constant conditions 
to become exactly $24.0 \mathrm{~h}$, indicating that Ta daily cycle entrained both $\mathrm{Tb}$ and LA rhythms. Indeed, an increase of the robustness was observed ranging from $27.1 \%$ and $7.3 \%$ under constant conditions to $58.3 \%$ and $15.3 \%$ under cycling Ta, respectively for Tb and LA rhythms (Tables 1 and 2).

In any case, when $\mathrm{Ta}$ rose, $\mathrm{Tb}$ gradually increased and its acrophase occurred during the heat phase, (Figures 4A-B, 5A-1 and 5B-1). For LA rhythm, actograms showed also high activity during heat phase while inactivity coincides with the cooling phase of Ta cycle (Figure 3, stage 3 and 4). Acrophases occurred at 15:39 $\pm 0.21 \mathrm{~h}$ for Tb rhythm and at 13:12 $\pm 0.12 \mathrm{~h}$ for LA rhythm (Tables 1 and 2).

The entrainment by the Ta cycles was confirmed by the fact that a $12-\mathrm{h}$ phase advance of its cycle (stage 4) led to a significant phase advance of the Tb and LA rhythms (Figures 3, 4A, 5A-2 and 5B-2). All the ten bucks of experiment 1 showed a clear entrainment of Tb rhythm by Ta cycles while in experiment 2 which was performed on 7 bucks, six exhibited an entrainment of LA rhythm by Ta cycles as can be shown in Figure 3; while only one animal displayed a weak response to the entrainment effect by this cue.

The shifting of the rhythm was only within $6 \pm 0.26$ days for Tb (Figures $5 \mathrm{~A}-2$ and $5 \mathrm{~B}-2$ ) while it took $10 \pm 0.26$ days for the LA (Figure 3). The final pattern of LA rhythm was completely synchronized to the new Ta cycle within $12.5 \pm 1.07$ days, having therefore a peak of activity during heat-phase at the subjective night. Accordingly, acrophase of LA occurred at $02: 49 \pm 0.27 \mathrm{~h}$ and that of $\mathrm{Tb}$ at $02: 20 \pm 0.16 \mathrm{~h}$ (Tables 1 and 2). The one-way ANOVA confirmed the existence of a significant $\left(\mathrm{Tb} \mathrm{F}_{(5.40)}=1114 ; P<0.0001\right.$ and $\left.\mathrm{LA} \mathrm{F}_{(2.50)}=874,9 ; P<0.0001\right)$ shifting in both acrophases.

When NRT and RT phases were separately analysed, LA onsets and offsets did not show statistical differences between successive days. This indicates a significant equality of the means around each Ta cycle transition (Figure 6). As for acrophase, the onset and the offset of LA were shifted and seemingly following the Ta cycle conditions (Figure 6). Indeed, the two parameters coincide respectively with the cold-heat and heat-cold transitions, whatever the nature of the Ta cycle. 
When the goats returned to the former Ta cycle with maximum of heat during subjective day (stage 5, experiment 1), (Figures 4 B, 5A-3 and 5B-3), the Tb rhythm was delayed and went back to its initial pattern observed in stage 3 . Acrophase was delayed by $12.56 \mathrm{~h}$ to occur at $14: 54 \pm 0.26 \mathrm{~h}$ (Table 1). The ascendant and descendant phases of $\mathrm{Tb}$ follow those of the Ta cycles as previously seen in stage 3 (Figure 4 and 5-A).

Concerning LA rhythm, a return from an inversed Ta cycle (stage 4) to the constant conditions (stage 5) induced a free-running state showing a daily delay of its phase (stage 5: Figure 3). Excluding the Ta cycling under DD conditions, the period becomes significantly different $\left(\mathrm{F}_{(4.30)}\right.$ $=39.23 ; P<0.0001)$ from $24.0 \mathrm{~h}$, precisely of $24.9 \pm 0.12 \mathrm{~h}$. The acrophase was also shifted to occur at $03: 35 \pm 0.32 \mathrm{~h}$. Moreover, the regularity of the rhythm decreased with a robustness that also dropped to $9.2 \pm 0.48 \%$ (Table 2).

All together, these changes emphasize once again the entrainment effect of Ta cycles.

From experiments 1 and 2, Ta seemed to be a zeitgeber capable of synchronizing the circadian clock of the goat. Even if two outputs of the clock (Tb, LA) are driven by Ta, as demonstrated previously, it remains critical to ascertain that these results correspond to a real entrainment of the circadian clock and not to a masking effect. So, we verified if Ta is able to entrain the endogenous rhythm of Mel, one of the most robust outputs of the clock activity of SCN. An endogenous rhythm of Mel under DD and constant Ta (stage 2) was observed in all animals (data not shown). No major changes were noticed in the Mel rhythm when Ta cycle with heating period during the subjective day was applied (stage 3). However, when daily Ta cycle was 12-h phase advanced (Maximum of Ta at the end of subjective night, Stage 4), the Mel rhythm was shifted (phase delay, Figure 7). A Mel phase advance was then observed when animals returned to the former Ta cycle with maximum of Ta during the subjective day (stage 5, Figure 7).

The paired t-test showed that Ta treatment induced significant phase differences for both onsets (Phi1) and offsets (Phi2) of Mel rhythm, except for offset in the return from RT (stage 4) to NRTr (stage 5) (Table 3). Under all these conditions, Mel rhythm peaked when the Ta cycle was in the cold-phase.

This article is protected by copyright. All rights reserved 


\section{Discussion}

The daily variations of $\mathrm{Tb}$ and LA in goat were previously recorded in several thermoregulatory ${ }^{39,40,41}$ or chrono-biological ${ }^{42,43,44,45}$ studies. They were clearly identified, but their circadian nature was not established yet, until we did in the present study. Indeed, our results demonstrate that both rhythms persist under constant conditions with a period close to $24.0 \mathrm{~h}$. This indicates that in the goat, as in other mammals $23,46,47,48,49,50,51$, Tb and LA comply with the rules defining circadian rhythms.

Under their desert biotope, goats are frequently exposed to a very wide range of daily Ta cycles. Such variations are suspected to strongly affect several systems and circuits in the brain, particularly, the circadian system as demonstrated in the camel ${ }^{23}$. Based on these specific findings, we have hypothesized that in the desert goat, cyclic fluctuations of Ta could also synchronize its master circadian clock. Our results clearly show that, under 24.0h applied DD, Ta cycle entrains the $\mathrm{Tb}$ and LA rhythms to exactly $24.0 \mathrm{~h}$. A 12 -h switch of the daily Ta cycle induced a clear shift of both rhythms. Similarly, the return to the former Ta cycle induced a few days later a return to the initial phase (phase delay) of the $\mathrm{Tb}$ rhythm. Such result raises a pertinent question regarding the interpretation of the observed effect: is it a real entrainment of the master clock or a masking effect of $\mathrm{Ta}$ on $\mathrm{Tb}$ and LA rhythms?

Zeitgebers are known to act on the period and phase of the rhythm ${ }^{18}$, while masking cues strengthen or weaken the rhythm and affect generally the amplitude and the shape of the overt rhythm $29,52,53$. A masking effect of $\mathrm{Ta}$ on $\mathrm{Tb}$ is unlikely since the observed $\mathrm{Tb}$ peak in the goat, as in the camel ${ }^{23}$, is delayed from that of Ta. This delay indicates that there is no important radiant heat load (radiant, conductive and convective) occurring at the moment of $\mathrm{Ta}$ peak. $\mathrm{Tb}$ is also modified by evaporative water loss, thermal conductance and metabolic heat production.

Moreover, in our study any significant heat production from locomotion during the experiment 1 is very unlikely since the LA of animals was restrained. The animals were also in perfect endothermy and any mechanisms of evaporative water loss, exogenous heat loading and dissipation are unlikely to occur.

When applying Ta cycle in experiment 2, a transient and progressive period before final synchronization of the LA rhythm is seen after several days. This transition is typical of an 
entraining effect on the circadian clock. Moreover, the observed changes in the period and phase led to infer that Ta does not induce the LA rhythm directly, but entrained it through a circadian clock. In natural conditions, the goat is a diurnal species ${ }^{34,44,54}$, showing its maximal daily activity during the heat phase (the day). During Ta entrainment, bucks were also active during the heat period, regardless of Ta cycle applied. The phase relationship of activity with Ta cycle, as seen in natural conditions, is therefore maintained under Ta entrainment. In all species studied until now, in which an entrainment of LA rhythm was shown, animals keep their phase relationship with Ta cycle, nocturnal species being active during the cold phase of Ta while diurnal species being active during the warm phase ${ }^{13,17,18,20,22,55}$.

The temperature range used in the present study (from cold: $16^{\circ} \mathrm{C}$ to heat: $32^{\circ} \mathrm{C}$ ) is seemingly strong enough to provide a temporal information for synchronizing LA rhythm of the goat. Similarly, close oscillations of Ta were able to induce an entrainment of LA rhythm in rodents ${ }^{18}$. It is also established that the thermo-neutral zone in the goat is between $13^{\circ} \mathrm{C}$ and $21^{\circ} \mathrm{C}^{56}$. We believe that in desert goat breeds, adapted to their biotope high temperatures, this interval would be higher. As previously reported, the used range of Ta cycle between $16^{\circ}$ to $32^{\circ} \mathrm{C}$ induced a stronger entrainment of LA rhythm. However, narrower ranges would probably provide a weaker zeitgeber. No attempt was made till now to investigate such intensity-response curve for temperature entrainment.

In mammals, Mel secretion from the pineal gland is known to be the most powerful phase-marker of the master circadian clock. It depends much more on LD than on Ta cycles ${ }^{8}$. In goat, as in the camel $^{23}$, under DD conditions, plasma Mel follows Ta cycle and its peak occurred only in the cryophase. Analysis of plasma Mel combined to Tb and LA under DD conditions has, therefore, confirmed that the daily Ta cycle entrain three outputs of the master circadian clock, which precludes any masking effect. Thus, Ta cycle is a true zeitgeber in the goat. Beside the camel, this finding constitutes the second demonstration of such clear entrainment of the circadian system by Ta cycles in mammalian species. How the non-photic information of Ta reaches the SCN through central and/or peripheral thermo-receptors, and how it could presumably change its molecular machinery still need further investigation.

This article is protected by copyright. All rights reserved 


\section{Conclusion}

The present work demonstrates that daily Ta cycle, a strong environmental cue in the desert habitat, is able to synchronize the central clock of the goat by entraining the Tb, LA and Mel rhythms. Until now, a similar effect has been described in only two mammalian species, namely the camel and herein the goat. This finding raises enquiry on whether such phenomenon represents common process of adaptation of the circadian system in heterothermic species to the desert or it is specific to these two species only. Accordingly, further investigations in other desert species are needed to elucidate the prevalence of this peculiar thermoregulatory feature in mammals.

\section{Acknowledgments}

The authors are grateful to Hassan II Agronomy and Veterinary Medicine Institute (Rabat, Morocco), the Institute of Cellular and Integrative Neurosciences (Strasbourg, France), the Laboratory of Hormonology (Lyon, France) and the Moroccan AVPN Association (Association de Vulgarisation et Promotion des Neurosciences) for supporting this study.

\section{Funding}

This study was funded by the International Foundation for Science (IFS: Research Grant Agreement No. I-3-B-6082-1).

\section{Conflict of Interest Statement}

The authors declare no actual or potential conflict of interest.

\section{Author Contributions}

Hicham Farsi: Performed the experiments; Analyzed and interpreted the data and drafted the manuscript. Driss Harti: Performed the experiments. Mohamed Rachid Achaâban: Analyzed and interpreted the data and drafted the manuscript. Mohammed Piro, Mohammed Ouassat, Véronique Raverot, Béatrice Bothorel: Contributed reagents, materials, analysis tools or data and drafted the manuscript. Paul Pévet, Etienne Challet: Conceived and designed the experiments; Analyzed and interpreted the data and drafted the manuscript. Khalid El Allali: Conceived and designed the

This article is protected by copyright. All rights reserved 
experiments; Analyzed and interpreted the data; Contributed reagents, materials, analysis tools or data and drafted the manuscript.

\section{References}

1. Refinetti R. Variability of diurnality in laboratory rodents. J Comp Physiol A Neuroethol Sens Neural Behav Physiol. 2006; 192(7):701-714.

2. Challet E. Minireview: Entrainment of the suprachiasmatic clockwork in diurnal and nocturnal mammals. Endocrinology. 2007; 148(12):5648-5655.

3. Dibner C, Schibler U, Albrecht U. The mammalian circadian timing system: organization and coordination of central and peripheral clocks. Annu Rev Physiol. 2010; 72:517-549.

4. Reppert SM, Weaver DR. Molecular analysis of mammalian circadian rhythms. Annu Rev Physiol. 2001; 63: 647-676.

5. Challet E, Pévet P. Interactions between photic and nonphotic stimuli to synchronize the master circadian clock in mammals. Front Biosci. 2003; 1:s246-257.

6. Takahashi JS, Hong HK, Ko CH, McDearmon EL. The genetics of mammalian circadian order and disorder: implications for physiology and disease. Nat Rev Genet. 2008; 9(10):764-775.

7. Mohawk JA, Green CB, Takahashi JS. Central and peripheral circadian clocks in mammals. Annu Review Neurosci. 2012. 35:445-462.

8. Rensing L, Ruoff P. Temperature effect on entrainment, phase shifting, and amplitude of circadian clocks and its molecular bases. Chronobiol Int. 2002; 19(5):807-864.

9. Brown SA, Zumbrunn G, Fleury-Olela, F, Preitner N, Schibler U. Rhythms of mammalian body temperature can sustain peripheral circadian clocks. Curr Biol. 2002; 12(18):15741583.

10. Tsuchiya Y, Akashi M, Nishida E. Temperature compensation and temperature resetting of circadian rhythms in mammalian cultured fibroblasts. Genes Cells. 2003; 8(8):713-720.

11. Buhr ED, Yoo SH, Takahashi JS. Temperature as a universal resetting cue for mammalian circadian oscillators. Science. 2010; 330(6002):379-385.

This article is protected by copyright. All rights reserved 
12. Hoffmann K. Die relative Wirksamkeit von Zeitgebem. Oecologia. 1969; 3:184-1969.

13. Tokura H, Aschoff J. Effects of Temperature on the Circadian Rhythm of PigTailed Macaques Macaca nemestrina. Am J Physiol. 1983(6); 245:R800-R804.

14. Aschoff J, Tokura H. Circadian activity rhythms in squirrel monkeys: entrainment by temperature cycles. J Biol Rhythms. 1986; 1(2):91-99.

15. Francis AJP, Coleman GJ. Ambient Temperature Cycles Entrain the Free Running Circadian Rhythms of the Stripe-Faced Dunnart, Sminthopsis macroura. J Comp Physiol A. $1990 ; 167(3): 357-362$.

16. Rajaratnam SM, Redman JR. Entrainment of activity rhythms to temperature cycles in diurnal palm squirrels. Physiol Behav. 1998; 63(2):271-277.

17. Pálková M, Sigmund L, Erkert HG. Effect of ambient temperature on the circadian activity rhythm in common marmosets, Callithrix j. jacchus (primates). Chronobiol Int. 1999; 16(2):149-161.

18. Refinetti R. Entrainment of circadian rhythm by ambient temperature cycles in mice. $J$ Biol Rhythms. 2010; 25(4):247-256.

19. Bouaouda H, Achaaban MR, Ouassat M, et al. Daily regulation of body temperature rhythm in the camel (Camelus dromedarius) exposed to experimental desert conditions. Physiol Rep. 2014; 2(9):e12151.

20. Lindberg RG, Hayden P. Thermoperiodic Entrainment of Arousal from Torpor in the Little Pocket Mouse, Perognathus longimembris. Chronobiologia. 1974 1(4):356-361.

21. Erkert HG, Rothmund E. Differences in Temperature Sensitivity of the Circadian Systems of Homoeothermic and Heterothermic Neotropical Bats. Comp Biochem Physiol. 1980; 68A:383-390.

22. Pohl H. Temperature Cycles as Zeitgeber for the Circadian Clock of Two Burrowing Rodents, the Normothermic Antelope Ground Squirrel and the Heterothermic Syrian Hamster. Biol Rhythm Res. 1998; 29, 311-325.

This article is protected by copyright. All rights reserved 
23. El Allali K, Achaaban, M.R., Bothorel, B., et al. Entrainment of the circadian clock by daily ambient temperature cycles in the camel (Camelus dromedarius). Am J Physiol Regul Integr Comp Physiol. 2013; 304(11):R1044-R1052.

24. Schmidt-Nielsen K, Schmidt-Nielsen B, Jarnum SA, Houpt TR. Body temperature of the camel and its relation to water economy. Am J Physiol. 1957; 188(1):103-112.

25. Cain JW, Krausman PR, Rosenstock SS, Turner JC. Mechanisms of thermoregulation and water balance in desert ungulates. Wildl Soc Bull. 2006; 34(3):570-581.

26. Mengistu U, Dahlborn K, Olsson K. Mechanisms of water economy in lactating Ethiopian Somali goats during repeated cycles of intermittent watering. Animal. 2007; 1(7):10091017.

27. Al-Tamimi H, Al-Atiyat R, Al-Majali A, Alameri O. Renal efficiency underlies adaptive heterothermy of heat-stressed hypohydrated goats. Trop Anim Health Pro. 2019; 51(8):2287-2295.

28. Touitou Y, Smolensky MH, Portaluppi F. Ethics, standards and procedures in human and animal research in chronobiology. Chronobiol Int. 2006; 23(6):1083-1096.

29. Refinetti R. The circadian rhythm of body temperature. Front Biosci. 2010; 15:564-594.

30. Piccione G, Caola G, Refinetti R. The circadian rhythm of body temperature of the Horse. Biol Rhythm Res. 2002; 33(1):113-119.

31. Lovegrove B. Modification and miniaturization of Thermochron iButtons for surgical implanation into small animals. J Comp Physiol B. 2009; 179 (4):451-458.

32. McCafferty DJ, Gallon S, Nord A. Challenges of measuring body temperatures of freeranging birds and mammals. Anim Biotelemetry. 2015; 3:33.

33. Adam D, Johnston S, Beard L, et al. Surgical implantation of temperature-sensitive transmitters and data-loggers to record body temperature in koalas (Phascolarctos cinereus). Aust Vet J. 2016; 94(1-2):42-7.

34. Farsi H, Harti D, Achaâban M R, et al. Validation of locomotion scoring as a new and inexpensive technique to record circadian locomotor activity in large mammals. Heliyon. 2018; 4(12):e00980.

This article is protected by copyright. All rights reserved 
35. Brun J, Chamba G, Khalfallah Y, et al. Effect of modafinil on plasma melatonin, cortisol and growth hormone rhythms, rectal temperature and performance in healthy subjects during a 36 h sleep deprivation. J Sleep Res. 1998; 7(2):105-14.

36. Vivien-Roels B, Pitrosky B, Zitouni M, et al. Environmental control of the seasonal variations in the daily pattern of melatonin synthesis in the European hamster, Cricetus cricetus. Gen Comp Endocrinol. 1997; 106(1):85-94.

37. Bothorel B, Barassin S, Saboureau M, et al. In the rat, exogenous melatonin increases the amplitude of pineal melatonin secretion by a direct action on the circadian clock. Eur $J$ Neurosci. 2002; 16(6):1090-1098.

38. El Allali K, Achaaban MR, Vivien-Roels B, Bothorel B, Tligui NS, Pévet P. Seasonal variations in the nycthemeral rhythm of plasma melatonin in the camel (Camelus dromedarius). J Pineal Res. 2005; 39(2):121-128.

39. Finch VA, Dmi'el R, Boxman R, Shkolnik A, Taylor CR. Why black goats in hot deserts? Effects of coat color on heat exchanges of wild and domestic goats. Physiol Zool. 1980; 53(1):19-25.

40. Shkolnik, A., Silanikove, N., Water economy, energy metabolism and productivity in desert ruminants. In: MorandFehr P Borbouse A \& De Simiane M eds., Nutrition and Systems of Goat Feeding, ITOVIC-INRA, Tours, France; 1981:236-246.

41. Maia AS, da Silva RG, Nascimento CC, Pedroza HP, Domingos HG. Thermoregulatory responses of goats in hot environments. Int J Biometeorol. 2015; 59(8):1025-1033.

42. Piccione G, Caola G, Refinetti R. Circadian rhythms of body temperature and liver function in fed and food-deprived goats. Comp Biochem Physiol A Mol Integr Physiol. 2003; 134(3):563-572.

43. Piccione G, Refinetti R. Thermal chronobiology of domestic animals. Front Biosci. 2003; $8: 258-264$.

44. Piccione G, Giannetto C, Assenza A., Fazio F, Caola G. Locomotor activity and serum tryptophan and serotonin in goats: Daily rhythm. J Appl Biomed. 2008; 6(1):73-79.

45. Piccione G, Giannetto C, Casella S, Caola G. Daily locomotor activity in five domestic animals. Animal Biol. 2010; 60(1):15-24.

This article is protected by copyright. All rights reserved 
46. Aschoff J. Human circadian rhythms in activity, body temperature and other functions. Life Sci Space Res. 1967; 5:159-173.

47. Stephan FK, Zucker I. Circadian rhythms in drinking behavior and locomotor activity of rats are eliminated by hypothalamic lesions. Proc Natl Acad Sci U S A. 1972; 69(6): 15831586.

48. Wever RA. Internal interactions within the human circadian system: the masking effect. Experientia, 1985; 41(3):332-342.

49. Pickard GE, Kahn R, Silver R. Splitting of the circadian rhythm of body temperature in the golden hamster. Physiol Behav. 1984; 32(5):763-766.

50. Kuwabara N, Seki K, Aoki K. Circadian, sleep and brain temperature rhythms in cats under sustained daily light-dark cycles and constant darkness. Physiol Behav. 1986; 38(2):283289.

51. Lehman MN, Silver R, Gladstone WR, Gibson M, Bittman EL. Circadian rhythmicity restored by neural transplant. Immunocytochemical characterization of the graft and its integration with the host brain. J Neurosci. 1987; 7(6):1626-1638.

52. Hanneman SK. Measuring circadian temperature rhythm. Biol Res Nurs. 2001; 2(4): 236248.

53. Kelly SG. Body temperature variability (Part 2): Masking influences of body temperature variability and a review of body temperature variability in disease. Altern Med Rev. (2007); 12(1):49-62.

54. El Allali K, Farsi H, Piro M, Achaaban RM, Ouassat M, Challet E, Pevet P.. Smartphone and a freely available application as a new tool to record locomotor activity rhythm in large mammals and humans. Chronobiol Int. 2019; 36(8): 1047-1057.

55. Fritsche P, Miethe G, Gattermann R. Circadian Activity Rhythm and Entrainment in the Subterranean Common Mole-Rat (Cryptomys hottentotus). Biol Rhythm Res. 1997; 28:116.

56. Martini, E. La termoregolazione. In: Fisiologia degli animali domestici Martini eds., Libreria Universitaria L. Tinarelli Bologna. (1973):1130.

This article is protected by copyright. All rights reserved 
Table 1: Parameters of the Tb rhythm during different stages of experiment 1.

\begin{tabular}{llcccc}
\hline Experiment 1 & Period & Acrophase & Mesor & Amplitude & Robustness \\
\hline Stage 1: LD uncontrolled Ta & $24.0 \pm 0.09 \mathrm{~h}^{\mathrm{a}}$ & $17: 23 \pm 0.28 \mathrm{~h}^{\mathrm{a}}$ & $38.74 \pm 0.12^{\circ} \mathrm{C}$ & $0.69 \pm 0.36^{\circ} \mathrm{C}$ & $40.0 \pm 2.7 \%$ \\
Stage 2: DD+CTa & $25.3 \pm 0.09 \mathrm{~h}^{\mathrm{b}}$ & $14: 55 \pm 0.14 \mathrm{~h}^{\mathrm{b}}$ & $38.82 \pm 0.06^{\circ} \mathrm{C}$ & $0.23 \pm 0.18^{\circ} \mathrm{C}$ & $27.1 \pm 1.8 \%$ \\
Stage 3: DD+NRT & $24.0 \pm 0.02 \mathrm{~h}^{\mathrm{a}}$ & $15: 39 \pm 0.21 \mathrm{~h}^{\mathrm{c}}$ & $39.18 \pm 0.10^{\circ} \mathrm{C}$ & $0.43 \pm 0.24^{\circ} \mathrm{C}$ & $58.3 \pm 3.0 \%$ \\
Stage 4: DD+RT & $24.0 \pm 0.02 \mathrm{~h}^{\mathrm{a}}$ & $02: 20 \pm 0.16 \mathrm{~h}^{\mathrm{d}}$ & $39.34 \pm 0.15^{\circ} \mathrm{C}$ & $0.55 \pm 0.38^{\circ} \mathrm{C}$ & $55.7 \pm 2.3 \%$ \\
Stage 5: DD+NRTr & $24.0 \pm 0.03 \mathrm{~h}^{\mathrm{a}}$ & $14: 54 \pm 0.26 \mathrm{~h}^{\mathrm{c}}$ & $39.18 \pm 0.18^{\circ} \mathrm{C}$ & $0.50 \pm 0.29^{\circ} \mathrm{C}$ & $57.3 \pm 1.9 \%$
\end{tabular}

Note: One-way ANOVA analysis was used for the calculation of statistical differences between periods and acrophases across different stages of the experiment 1. Different superscript lowercase letters indicate significant differences between different stages ( $\leq \leq 0.05)$. Similar superscript lowercase letters indicate no statistically significant differences between compared stages $(\mathrm{p}>0.05)$. 
Table 2: Parameters of the LA rhythm during different stages of experiment 2.

\begin{tabular}{lccccc}
\hline Experiment 2 & Period & Acrophase & Mesor & Amplitude & Robustness \\
\hline Stage 1: LD uncontrolled Ta & $24.0 \pm 0.04 \mathrm{~h}^{\mathrm{a}}$ & $12: 33 \pm 0.30 \mathrm{~h}^{\mathrm{a}}$ & $1.59 \pm 0.08^{\circ} \mathrm{C}$ & $1.70 \pm 0.09^{\circ} \mathrm{C}$ & $32.0 \pm 1.8 \%$ \\
Stage 2: DD+CTa & $25.0 \pm 0.10 \mathrm{~h}^{\mathrm{b}}$ & $10: 58 \pm 0.30 \mathrm{~h}^{\mathrm{aA}}$ & $1.20 \pm 0.90^{\circ} \mathrm{C}$ & $0.60 \pm 0.02^{\circ} \mathrm{C}$ & $07.3 \pm 0.3 \%$ \\
Stage 3: DD+NRT & $24.0 \pm 0.05 \mathrm{~h}^{\mathrm{a}}$ & $13: 12 \pm 0.12 \mathrm{~h}^{\mathrm{aAb}}$ & $1.60 \pm 0.06^{\circ} \mathrm{C}$ & $1.00 \pm 0.03^{\circ} \mathrm{C}$ & $15.3 \pm 1.3 \%$ \\
Stage 4: DD+RT & $24.0 \pm 0.03 \mathrm{~h}^{\mathrm{a}}$ & $02: 49 \pm 0.27 \mathrm{~h}^{\mathrm{bAcB}}$ & $1.53 \pm 0.10^{\circ} \mathrm{C}$ & $0.86 \pm 0.07^{\circ} \mathrm{C}$ & $16.4 \pm 1.1 \%$. \\
Stage 5: DD+CTa & $24.9 \pm 0.12 \mathrm{~h}^{\mathrm{b}}$ & $03: 35 \pm 0: 32 \mathrm{~h}^{\mathrm{cAdB}}$ & $1.31 \pm 0.06^{\circ} \mathrm{C}$ & $0.47 \pm 0.10^{\circ} \mathrm{C}$ & $09.2 \pm 0.5 \%$
\end{tabular}

Note: One-way ANOVA analysis was used for the calculation of the statistical differences between periods and acrophases across different stages of the experiment 2. Different superscript lowercase or uppercase letters indicate significant differences between different stages $(\mathrm{p} \leq 0.05)$. Similar superscript lowercase or uppercase letters indicate no statistically significant differences between compared stages ( $p>0.05)$.

This article is protected by copyright. All rights reserved 
Table 3: Mean values \pm S.E.M. of individual phi1 and phi2 estimated with the nonlinear regressions applied for each animal in NRT, RT and NRTr conditions of Ta cycle respectively during stages 3,4 and 5 .

\section{Phi 1}

\begin{tabular}{ccc}
\hline NRT & RT & NRTr \\
(Stage 3) & $($ Stage 4) & (Stage 5) \\
\hline $16,57 \pm 1,80^{\mathrm{a}}$ & $13,21 \pm 2,07^{\mathrm{bA}}$ & $13,99 \pm 2,29^{\mathrm{B}}$
\end{tabular}

\section{Phi 2}

\begin{tabular}{ccc}
\hline NRT & RT & NRTr \\
$($ Stage 3) & $($ Stage 4) & (Stage 5) \\
\hline $10,24 \pm 1,65^{\mathrm{a}}$ & $14,17 \pm 1,94^{\mathrm{bA}}$ & $8,96 \pm 2,02^{\mathrm{A}}$
\end{tabular}

Note: Paired t-test analysis for the estimated Phi1 and Phi2 were performed for NRT and RT and then for RT and NRTr treatments: 1) Different superscript lowercase letters indicate significant differences between NRT and RT treatment for both Phi1 and Phi2 $(P \leq 0.05) .2)$ Different superscript uppercase letters indicate significant differences between RT and NRTr treatment in Phi1 $(P \leq 0.05)$. 3). Similar superscript uppercase letters indicate no statistically significant differences between RT and NRTr treatment in Phi2 (p>0.05).

This article is protected by copyright. All rights reserved 


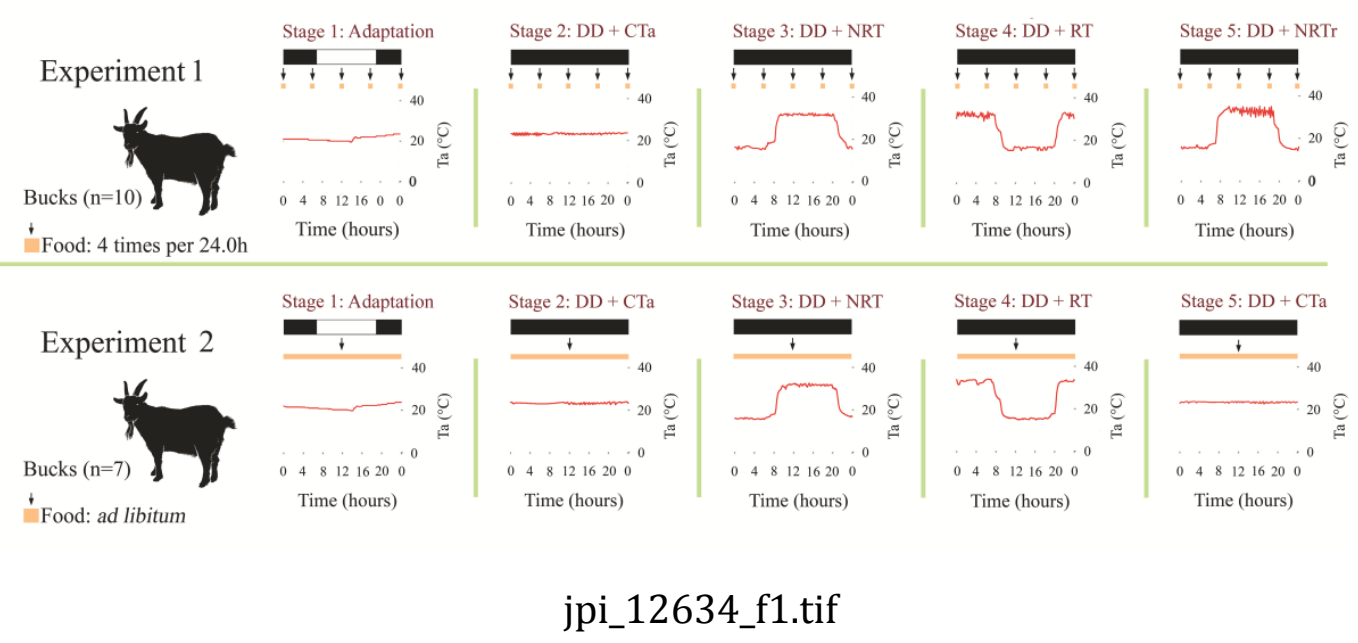

This article is protected by copyright. All rights reserved 


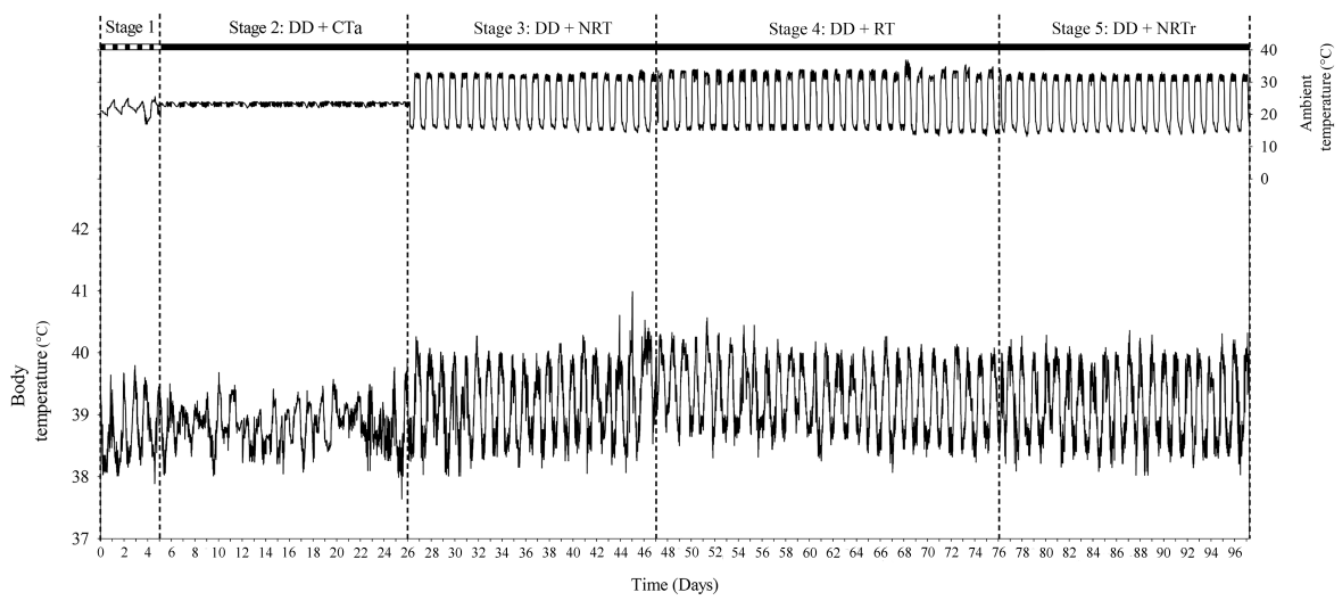

jpi_12634_f2.tif 


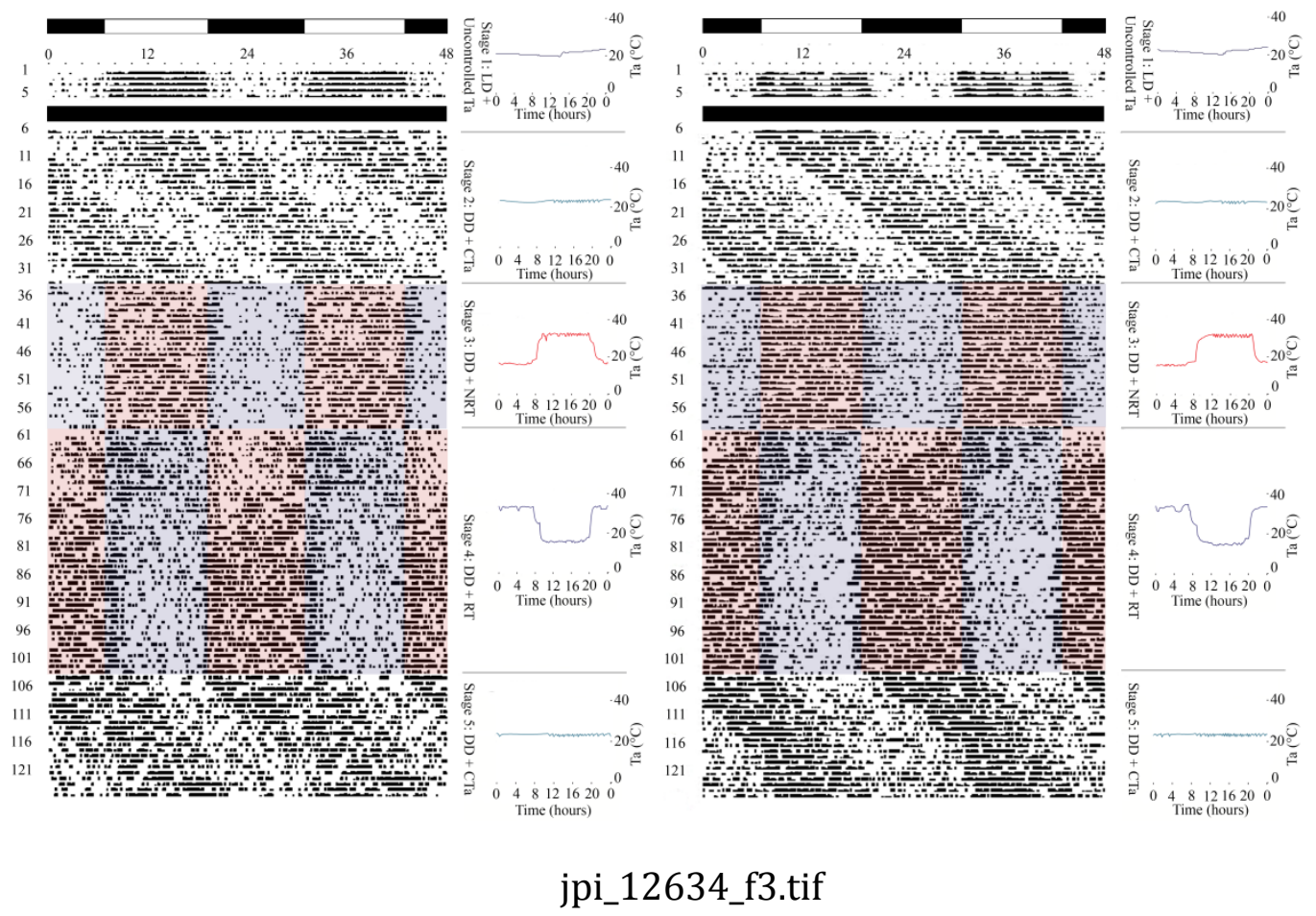

This article is protected by copyright. All rights reserved 

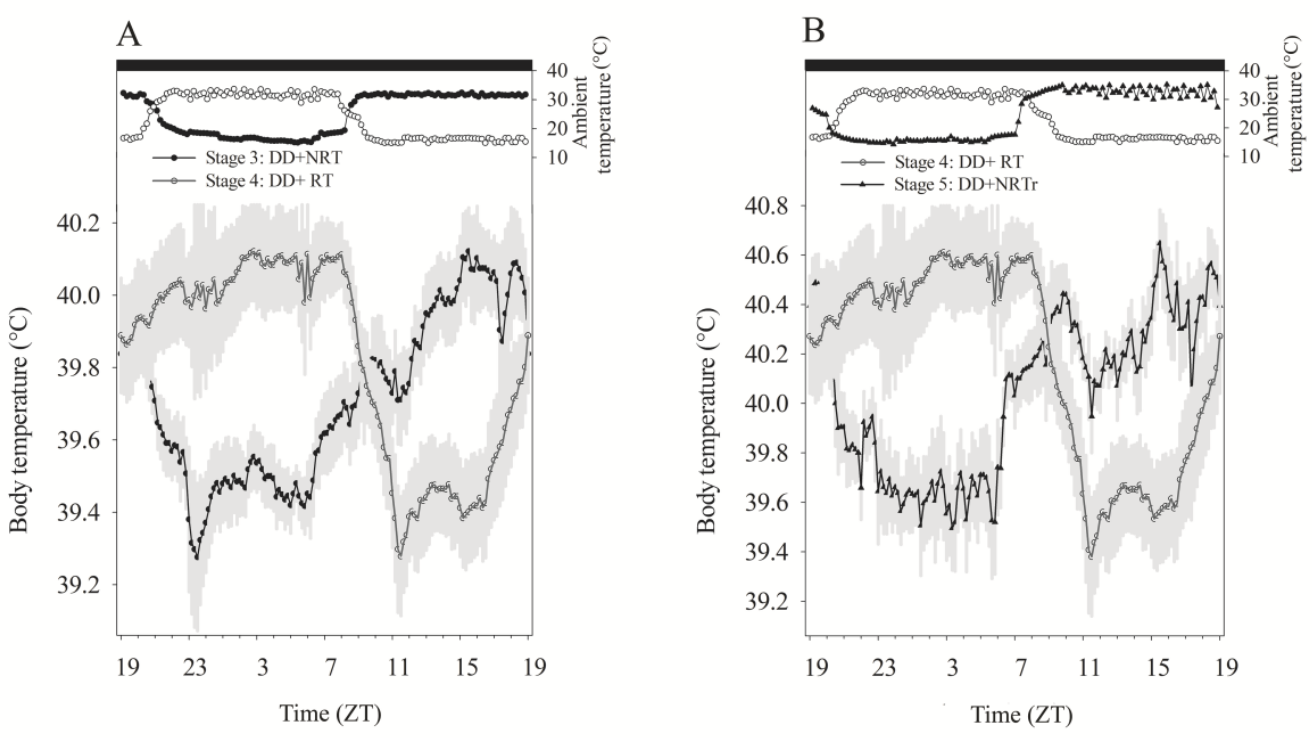

jpi_12634_f4.tif 
A-1

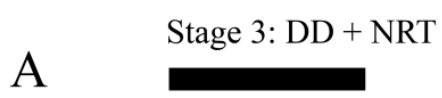

A Stage 3: DD + NRT

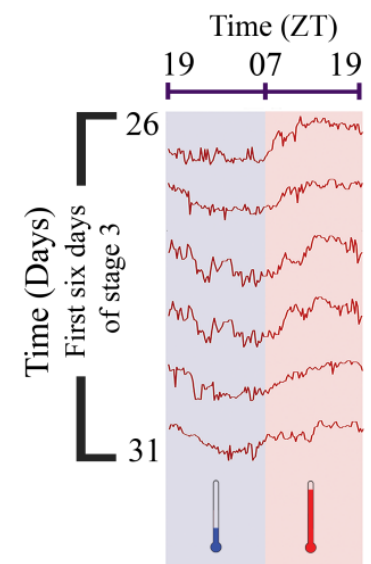

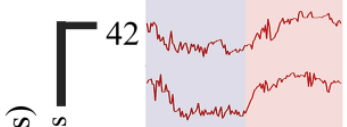

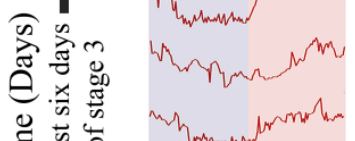

的范

E

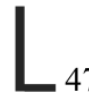

47
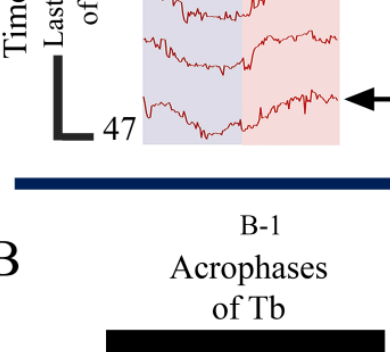

$\begin{array}{lllllll}00 & 04 & 08 & 12 & 16 & 20 & 00\end{array}$

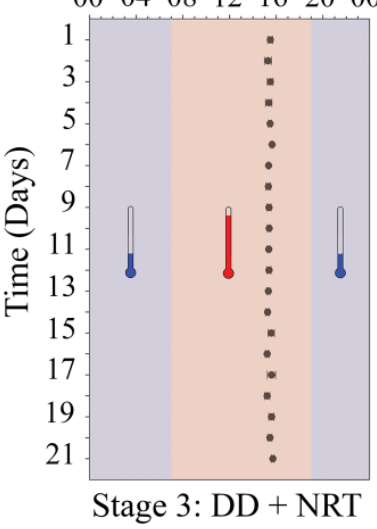

A-2

Stage 4: DD + RT
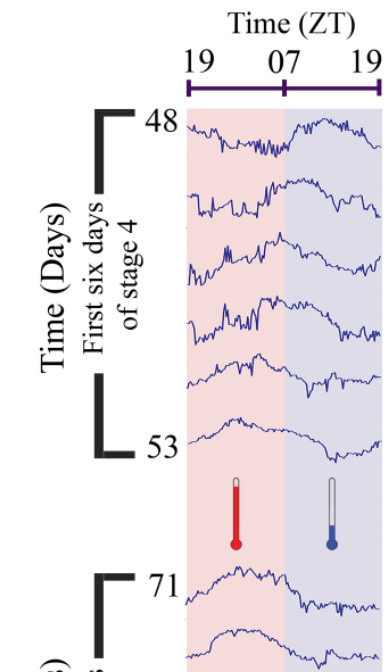

क

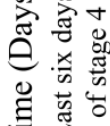
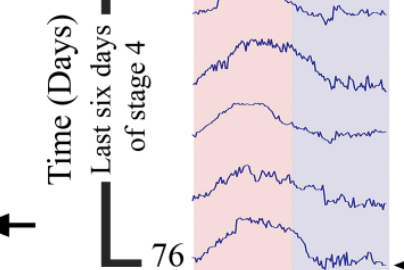

B-2

Acrophases

of $\mathrm{Tb}$

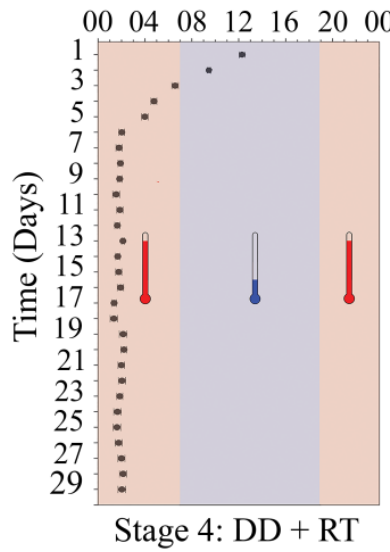

jpi_12634_f5.tif
A-3

Stage 5: DD + NRTr
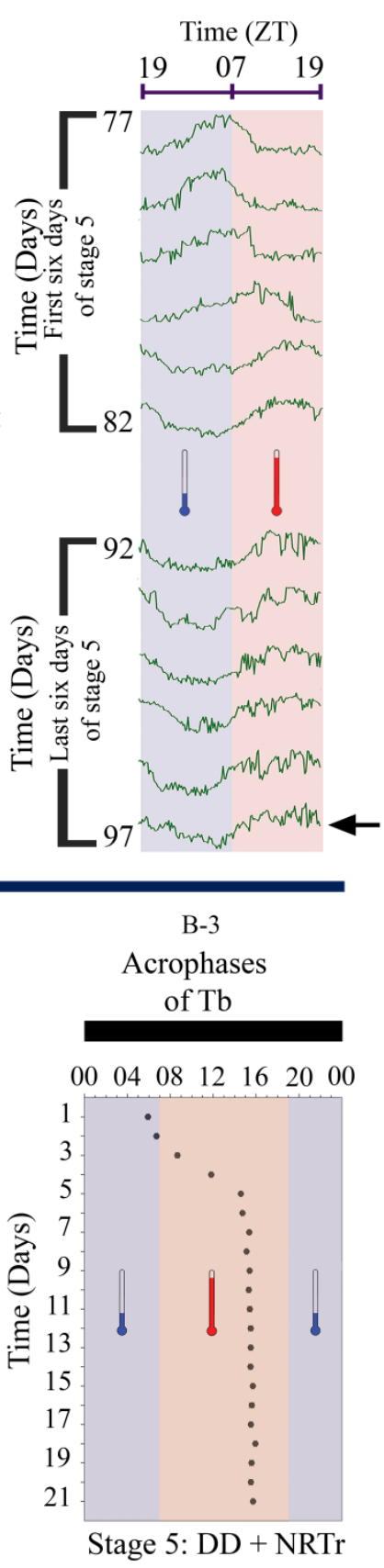

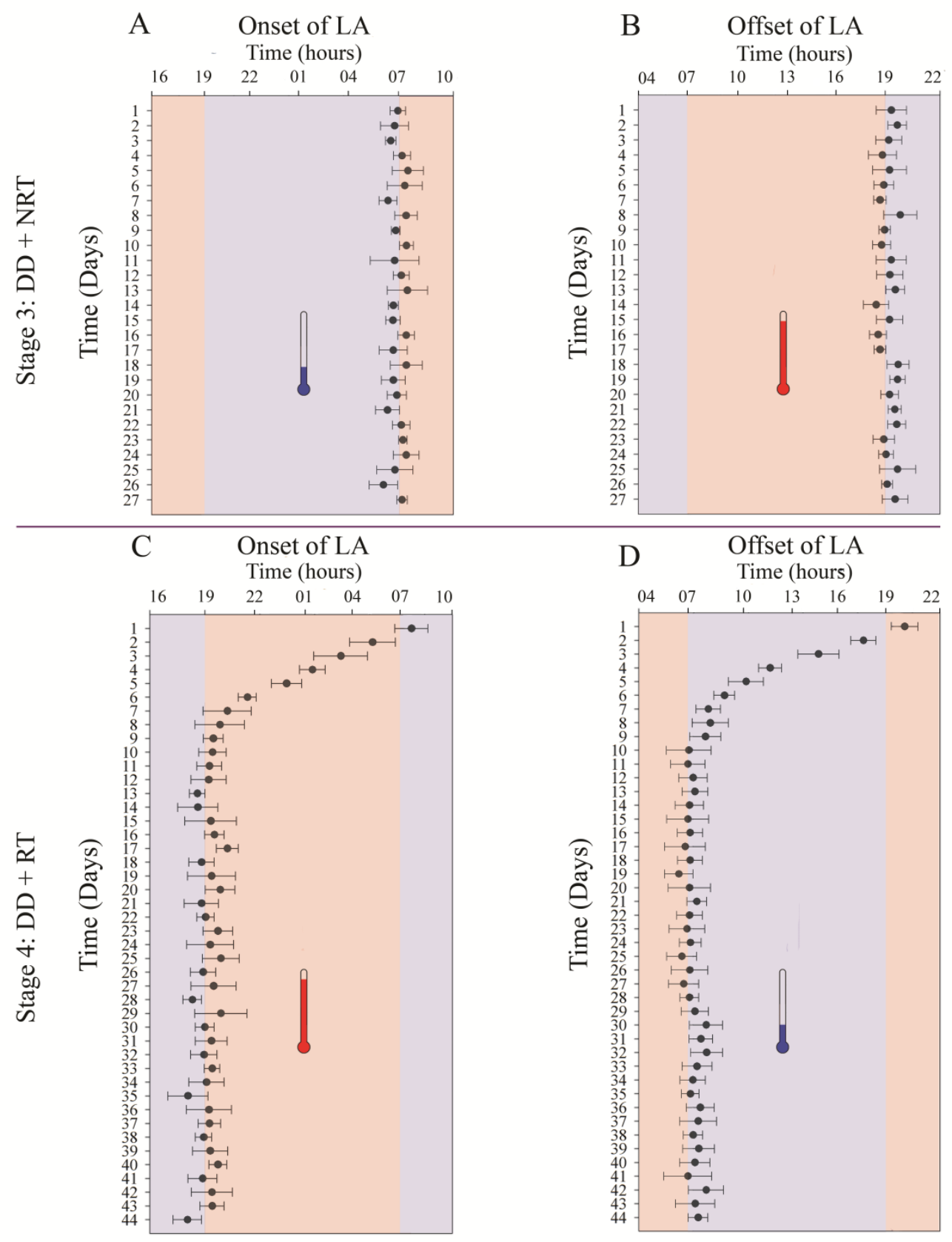

jpi_12634_f6.tif 

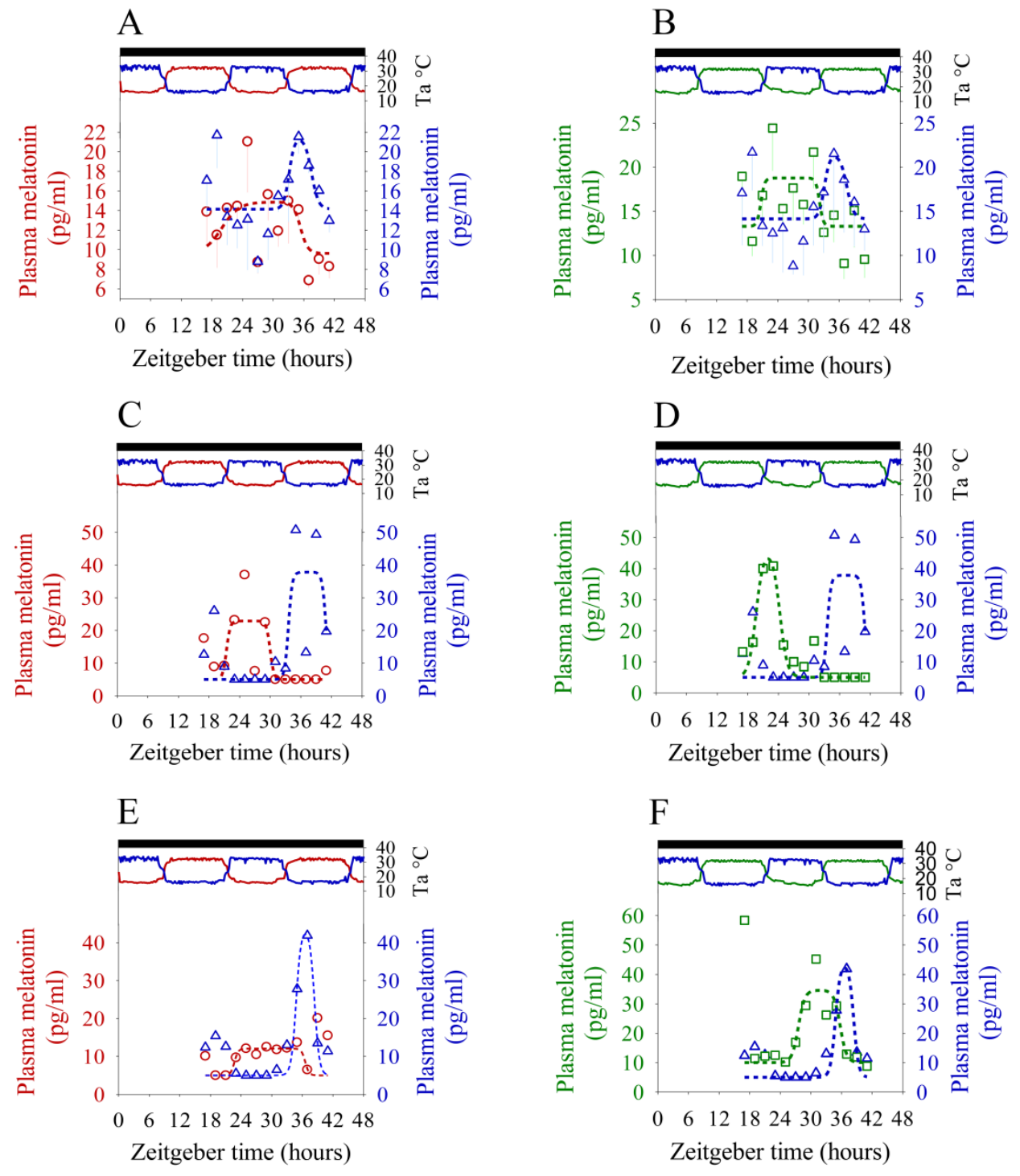

jpi_12634_f7.tif 